\title{
GENERALIZED HERMITE POLYNOMIALS OBTAINED BY EMBEDDINGS OF THE $q$-HEISENBERG ALGEBRA
}

\author{
JOACHIM SEIFERT \\ Sektion Physik, Universität München, LS Prof. Wess \\ Theresienstr. 37, D-80333 München, Germany \\ E-mail: Joachim.Seifert@physik.uni-muenchen.de \\ WWW: www.ls-wess.physik.uni-muenchen.de/ seifert
}

\begin{abstract}
Several ways to embed $q$-deformed versions of the Heisenberg algebra into the classical algebra itself are presented. By combination of those embeddings it becomes possible to transform between $q$-phase-space and $q$-oscillator realizations of the $q$-Heisenberg algebra. Using these embeddings the corresponding Schrödinger equation can be expressed by various difference equations. The solutions for two physically relevant cases are found and expressed as Stieltjes Wigert polynomials.
\end{abstract}

\section{Introduction}

1.1. The q-Heisenberg algebra in phase space variables. In a classical paper [1] J. Wess and J. Schwenk introduced a deformed version of the Heisenberg algebra, where both the deformed momentum and position operators can be represented by self-adjoint operators on a Hilbert-Space. The so called toy model-algebra [1] reads:

$$
\begin{gathered}
\sqrt{q} \xi p-\frac{1}{\sqrt{q}} p \xi=i u \\
\frac{1}{\sqrt{q}} \xi p-\sqrt{q} p \xi=i u^{*} \\
u p=q p u \quad u \xi=q^{-1} \xi u \quad u u^{*}=u^{*} u=1,
\end{gathered}
$$

where $q>1, q \in R$ is the deformation parameter. In [2] the following quantum-mechanical representation is obtained:

$$
p\left| \pm P_{n}>= \pm \pi_{0} q^{n}\right| \pm P_{n}>\quad x\left| \pm X_{k}>= \pm \frac{1}{\pi_{0 \sqrt{q}\left(q-q^{-1}\right)}} q^{k}\right| \pm X_{k}>
$$

Here either $\mid \pm P_{n}>$ or $\mid \pm X_{n}>$ ( for one value of + or - ) form an abstract basis for

1991 Mathematics Subject Classification: Primary 81R50; Secondary 20 C35.

The paper is in final form and no version of it will be published elsewhere. 
an irreducible representation of the $q$-Heisenberg algebra. This representation is physical in the sense that all real elements of the $*$ algebra $\left(^{1}\right)$ are represented by essentially self-adjoint operators. Therefore it is necessary to consider two copies $\left(\mid \pm P_{n}>\right.$ for + or -) of irreducible representations [2] of $p$ to have $\xi$ act as an essentially self-adjoint operator $\left({ }^{2}\right)$. The dual argument is valid for $x$ and $\mid \pm X_{k}>$. As in the classical case a transformation between $\mid \pm P_{n}>$ and $\mid \pm X_{n}>$ exists, and is given by the $q$-Fourier transformation.

1.2. The q-Heisenberg algebra in oscillator variables. In the classical case a very useful parameterization of the Heisenberg algebra can be given by annihilation and creation operators. Recently $q$-deformations of this oscillator representation have been discussed by many authors $[7,8,10]$. Macfarlane in an early paper [7] proposed the following $q$ oscillator :

$$
a_{q} a_{q}^{*}-q^{-2} a_{q}^{*} a_{q}=1
$$

This particular parameterization is very convenient, as the number-operator $N$ does not explicitly appear in the commutation relations. The Casimir operator that is present in more general $q$-oscillator algebras [8] is here already fixed to the particular value of 0 . As a result only Fock type Hilbert space representations exist. Those have the general form:

$$
a_{q} a_{q}^{*}\left|n>_{q}=(n)_{q^{-2}}\right| n>_{q} \quad(n)_{q^{-2}}:=\frac{1-q^{-2 n}}{1-q^{-2}}=:\{n\}_{q}
$$

Contrarily to the classical case a transformation between the two sets of operators $(p, \xi$ versus $a_{q}, a_{q}^{*}$ ) is far from trivial. A possible solution is to embed the $q$-deformed algebras into the classical ones, perform the transformation there and go back to the $q$-case afterwards .

\section{Embeddings of the algebras}

2.1. In oscillator variables. By a general theorem it is possible to embed the $q$ algebras into the universal-enveloping algebras of the classical Lie algebras $\left({ }^{3}\right)$

Curtwright and Zachos considered these matters thoroughly in [9] and found the following map relating the classical and the $q$-oscillator:

$$
a_{q}^{*}=\sqrt{\frac{[N]}{N}} a^{*} \quad a_{q}=a \sqrt{\frac{[N]}{N}}
$$

It is obvious that this is a valid embedding: The deformed creator creates the classical state, takes out the undeformed occupation number content and multiplies with the $q$ deformed boson number. Therefore the states coincide, and the Hilbert space of the $q$-oscillator is identical to the one of the undeformed oscillator.

\footnotetext{
$\left({ }^{1}\right)$ i.e. the observables!

$\left({ }^{2}\right)$ By extending the domain of $\xi$ one shrinks the domain of $\xi^{\dagger}$.

$\left.{ }^{3}\right)$ Such transformations always exist as long as one is only concerned with the algebra part of it. For Bi- or Hopf-algebras the existence of such transformations is far from trivial.
} 
Another algebraically simpler embedding was found by the author [11] :

$$
\begin{aligned}
& a_{q}=\frac{e^{i \Phi}}{\sqrt{q^{2}-1}} e^{\sqrt{2} h a^{*}}\left\{q^{s}-e^{-\sqrt{2} h a}\right\} \\
& a_{q}^{*}=\frac{e^{i \Phi}}{\sqrt{q^{2}-1}}\left\{q^{\bar{s}}-e^{-\sqrt{2} h a^{*}}\right\} e^{\sqrt{2} h a}
\end{aligned}
$$

Both embeddings lead to the $q$-oscillator relations [7]:

$$
a_{q} a_{q}^{*}-q^{-2} a_{q}^{*} a_{q}=1
$$

if $q=e^{h^{2}}$. In the second case the $q$-oscillator states explicitly differ from the classical ones (although they still lie in the same Hilbert-space). An arbitrary complex constant has been introduced in the equations $(7,8)$, together with an arbitrary phase factor - both label a certain arbitrariness in the definition of the embedding $\left({ }^{4}\right)$. From the algebraic form of the embedding, it is obvious that $a_{q}$ will tend to the classical $a$ for $q \rightarrow 1$. In fact the following expansion in $h=\sqrt{\log (q)}$ exists:

$$
a_{q}=\gamma(h) \sum_{n=0}^{\infty} \frac{(\sqrt{2} h)^{n}}{n !} \cdot\left\{\sum_{j=0}^{n} \frac{n !}{(n+1-j) ! j !}(-)^{n+1-j} a^{* j} a^{n-j}\right\} \cdot a
$$

In (9) the regular part of the normalization constant has been separated:

$$
\frac{1}{\sqrt{q^{2}-1}}=\underbrace{\frac{\sqrt{2} h}{\left(e^{2 h^{2}}-1\right)^{\frac{1}{2}}}}_{=: \gamma(h)} \cdot \frac{1}{\sqrt{2} h}
$$

An expansion of $\gamma(h)$ can be calculated involving a generalization of the Bernoulli numbers.

2.2. In phase space variables. The model of Wess and Schwenk can also easily be embedded by the following relations, that are motivated by the form of the spectrum of $\xi$ and $p$ :

$$
\begin{aligned}
& \xi=e^{h \hat{X}} \quad u=e^{-i h \hat{P}} \\
& p=i e^{-h \hat{X}} \frac{q^{\frac{1}{2}} e^{-i h \hat{P}}-q^{-\frac{1}{2}} e^{i h \hat{P}}}{q-q^{-1}}
\end{aligned}
$$

Here $\hat{X}$ and $\hat{P}$ denote the generators of the classical Heisenberg algebra, with $[\hat{X}, \hat{P}]=i$ A whole set of such transformations can be constructed because of the relation: $e^{h_{1} \hat{X}} e^{-i h_{2} \hat{P}}=e^{h_{1} h_{2}} e^{-i h_{2} \hat{P}} e^{h_{1} \hat{X}}$ which can be mapped to $u p=q p u$ or $u \xi=q^{-1} \xi u$ in several ways. Basically any hermitian operator and any unitary operator, that permute up to a factor can be used for a realization of the toy model algebra.

As for the spectrum it might not be obvious that the embedding (11) has the right classical limit. One has to keep in mind that the classical operators can arbitrarily be

$\left({ }^{4}\right)$ As one easily sees this freedom is related to the translational symmetry of the classical $\hat{X}$ and $\hat{P}$, that results in a complex translational invariance of $a$. 
shifted, and rescaled without changing the commutation relations $\left(^{5}\right)$. For the further considerations this problem will not be relevant, as only differences of those operators will appear, so that the diverging constants cancel, and will not appear in the limit.

Recently Julius Wess and Moritz Fichtmueller found another possible embedding [3] by studying the Casimir operator of the Curtwright Zachos map for $S U_{q}(1,1)$. This embedding has a sane behavior for $q \rightarrow 1$, in fact the classical momentum operator $\hat{P}$ remains undeformed. The explicit form of the embedding is:

$$
\begin{aligned}
& p=\hat{P} \quad \xi=\frac{\left[z+\frac{1}{2}\right]}{z+\frac{1}{2}} \hat{X} \\
& u=q^{z}
\end{aligned}
$$

Here the virial operator $z$ has been introduced by:

$$
z=-\frac{i}{2}(\hat{X} \hat{P}+\hat{X} \hat{P})
$$

The implications of this alternative embedding for the scheme presented here are under investigation now [11].

2.3. The scheme to find a transformation. Performing the following steps:

$$
\left(a_{q}, a_{q}^{*}\right) \Longleftrightarrow\left(a, a^{*}\right) \stackrel{\text { trivially }}{\Longleftrightarrow}(\hat{X}, \hat{P}) \Longleftrightarrow(\xi, p)
$$

one can find a whole set of $q$-oscillator relations, i.e. functions $a(\xi, p, u)$ and $a^{*}(\xi, p, u)$, adapted to the geometry of the exponential lattice on the $q$-deformed real line. A particular simple one is found to be:

$$
\begin{aligned}
& a_{q}=\frac{1}{\sqrt{1-q^{-2}}} u\left\{u-\xi q^{s-\frac{1}{2}}\right\} \\
& a_{q}^{*}=\frac{1}{\sqrt{1-q^{-2}}}\left\{u^{*}-\xi q^{\bar{s}-\frac{1}{2}}\right\} u^{*}
\end{aligned}
$$

One difference with the classical case is immediately seen. In the classical case $a$ is invariant under the classical Fourier transformation, in the quantum-case the $q$-Fouriertransform will get us to another realization of the $a_{q}$. A repeated application of the Fourier transform brings us back again to $a_{q}$. In fact by using the Fourier transformed annihilator for $(15) b_{q}=\frac{1}{\sqrt{1-q^{-2}}} u^{*}\left\{u^{*}-p q^{s-\frac{1}{2}}\right\}$ one can explicitly calculate a nontrivial example of a $q$ - Fourier transform.

3. Discussion of the model. In a first step the eigenvectors of the $q$-oscillator will be explicitly constructed in terms of the non-deformed eigenstates. In the next section those general relations will be applied to two different Hilbert space representations of the problem. The condition that fixes the groundstate reads:

$$
a_{q}\left|0>_{q}=0 \quad \rightarrow e^{-\sqrt{2} h a}\right| 0>_{q}=q^{s} \mid 0>_{q}
$$

$\left({ }^{5}\right)$ The translation symmetry is lost in the $q$-case. In fact shift and scale symmetry change to scale and exponential symmetry in $q$-quantum mechanics. That's basically why multi-particle systems are hard to define. 
The $q$-deformed groundstate is thus an ordinary coherent-state $\mid z>$ with $z=-\frac{h}{\sqrt{2}} s$. Explicitly we have:

$$
\left|0>_{q}=q^{-\frac{|s|^{2}}{4}} \cdot e^{-\frac{h s}{\sqrt{2}} a^{*}}\right| 0>\quad \Leftrightarrow \quad a\left|0>_{q}=-\frac{h s}{\sqrt{2}}\right| 0>_{q}
$$

For the excited states we find the following expression:

$$
\left|n>_{q}=\frac{q^{-n}}{\sqrt{\left(q^{-2} ; q^{-2}\right)_{n}}}\left(q^{-\bar{s}} e^{-\sqrt{2} h a^{*}} ; q^{-2}\right)_{n}\right| 0>_{q}
$$

where $(a ; q)_{n}:=(1-a)(1-a q) \ldots\left(1-a q^{n-1}\right)$ denotes the $q$-shifted factorial. As the factor $\frac{q^{-n}}{\sqrt{\left(q^{-2} ; q^{-2}\right)_{n}}}$ has a pole of the order $h^{-n}$ it might seem that this expression is not regular, but as the second term has by construction an n-fold zero with $h^{n}$ the singularities cancel and the expression is well behaved. An explicit formula can be obtained by expanding the $q$-factorial:

$$
\left|n>_{q}=\frac{q^{-n}}{\sqrt{\left(q^{-2} ; q^{-2}\right)_{n}}} \sum_{k=0}^{n}(-)^{k} q^{-k(k-1)}\left(\begin{array}{l}
n \\
k
\end{array}\right)_{q^{-2}} q^{-k \bar{s}} e^{-\sqrt{2} h k a^{*}}\right| 0>_{q}
$$

It can be shown that the case of $s \neq 0$ can be derived from the case with $s=0$ by a unitary transformation. (These are the remnants of the classical translational invariance, as can be seen by using the embedding (11) of the Heisenberg-algebra).

$$
\begin{aligned}
& \left|n ; s>_{q}=e^{\frac{h}{\sqrt{2}}\left(a \bar{s}-a^{*} s\right)} \cdot \frac{q^{-n}}{\sqrt{\left(q^{-2} ; q^{-2}\right)_{n}}}\left(e^{-\sqrt{2} h a^{*}} ; q^{-2}\right)_{n}\right| 0> \\
& \left|n ; s>_{q}:=\hat{\mathcal{D}}(s)\right| n ; s=0>_{q}
\end{aligned}
$$

Here the right hand side is relative to the undeformed groundstate, i. e. a coherentstate with $a$-eigenvalue 0 . We are therefore able by (21) to generated a whole family (indexed by a complex-parameter s) of solutions to the $q$-oscillator problem. Because of the transformation $\hat{\mathcal{D}}(s)$ we will restrict ourselves to the case of $s=0$ from now on:

4. Construction of the eigenstates. From (19) one sees that the eigenstates of the $q$-oscillator can be written as superpositions of ordinary coherent states:

$$
\begin{aligned}
& \left|n ; s=0>_{q}=\frac{q^{-n}}{\sqrt{\left(q^{-2} ; q^{-2}\right)_{n}}} \sum_{k=0}^{n}(-)^{k} C_{k}^{n}\left(q^{-2}\right) e^{-\sqrt{2} h k a^{*}}\right| 0> \\
& =\frac{q^{-n}}{\sqrt{\left(q^{-2} ; q^{-2}\right)_{n}}} \sum_{k=0}^{n}(-)^{k} q^{k}\left(\begin{array}{l}
n \\
k
\end{array}\right)_{q^{-2}} \mid-\sqrt{2} h k>
\end{aligned}
$$

Here the $C_{k}^{n}\left(q^{-2}\right):=q^{-k(k-1)}\left(\begin{array}{l}n \\ k\end{array}\right)_{q^{-2}}$ are a special deformation of the binomial-coefficients, satisfying : $C_{k}^{n+1}(q)=C_{k}^{n}(q)+q^{n} C_{k-1}^{n}(q)$.

4.1. Classical position representation. It is instructive to investigate the $q$-deformed states in the usual (undeformed) position representation. We will see that contrarily to the undeformed case the solution does not factor into a polynomial and the ground-state, but we find a set of functions in $q$ (and $s$ ), orthogonal with respect to the classical measure, that tend to the Hermite-polynomials as $h \rightarrow 0$. 
The groundstate is given by:

$$
<x \mid 0>=\pi^{-\frac{1}{4}} e^{-\frac{1}{2} x^{2}}
$$

and satisfies:

$$
<x|-\sqrt{2} h k>=<x| e^{i 2 h k P}|0>=<x+2 h k| 0>
$$

putting this into (22) and using the generating function for the Hermite polynomials:

$$
\sum_{n} \frac{s^{n}}{n !} H_{n}(z)=e^{-s^{2}+2 s z}
$$

The following equation is obtained:

$$
\begin{aligned}
& <x\left|n>_{q}=\frac{q^{-n}}{\sqrt{\left(q^{-2} ; q^{-2}\right)_{n}}} \sum_{k=0}^{n}(-)^{k} q^{k}\left(\begin{array}{l}
n \\
k
\end{array}\right)_{q^{-2}} e^{-2 k^{2} h^{2}-2 k h x} \cdot<x\right| 0> \\
& =\frac{q^{-n} n !}{\sqrt{\left(q^{-2} ; q^{-2}\right)_{n}}} \sum_{j=0} \mathbf{S}_{j}^{(n)}\left(q^{-2}\right) \frac{(-h)^{j}}{j !} H_{j}(x) \cdot<x \mid 0>
\end{aligned}
$$

Here the notion of a $q$-deformed Stirling number has been introduced:

$$
\begin{aligned}
& n ! \cdot \mathbf{S}_{j}^{(n)}(q):=\left.(-)^{n}\left(\frac{d}{d x}\right)^{j}\left(e^{x} ; q\right)_{n}\right|_{x=0} \\
& =(-)^{n}\left(\frac{d}{d x}\right)^{j} \prod_{k=0}^{n-1}\left(1-q^{k} e^{x}\right)=\sum_{k=0}^{n}(-)^{n-k} C_{k}^{n}(q) k^{j}
\end{aligned}
$$

Which take the special values:

$$
\mathbf{S}_{0}^{(n)}(q)=0 \quad \mathbf{S}_{1}^{(n)}(q)=\frac{1}{n !}(-)^{n-1}(q ; q)_{n-1} \quad \mathbf{S}_{n}^{(n)}(q)=q^{\frac{n(n-1)}{2}}
$$

To get the correct classical limit one has to cancel out the zeros and poles of the two factors. First one isolates the regular part of the normalization factor $\gamma(q)$. Using $q=e^{h^{2}}$ one obtains:

$$
\gamma(q):=\frac{q^{-n}}{\sqrt{\left(q^{-2} ; q^{-2}\right)_{n}}}=\frac{1}{\sqrt{(n)_{q^{-2}} !}} \cdot \underbrace{\left(\frac{2 h^{2}}{e^{2 h^{2}}-1}\right)^{\frac{n}{2}}}_{:=\gamma\left(h^{2}\right)} \cdot \frac{1}{\sqrt{2^{n}}} \cdot h^{-n}
$$

Then one extracts the zeros of the $q$-Stirling numbers:

$$
\mathcal{S}_{k}^{(n)}\left(h^{2}\right):=\mathbf{S}_{k}^{(n)}(q) \cdot h^{-2(n-k) \cdot \theta(n-k)}
$$

Those new numbers $\mathcal{S}_{k}^{(n)}(q)$ do not tend versus the classical Stirling-numbers for $q \rightarrow 1$ if $k<n$, but they are convenient to write down the expansion:

$$
\begin{aligned}
& <x \mid n>_{q}=\frac{1}{\sqrt{2^{n}(n)_{q^{-2}} !}} \gamma\left(h^{2}\right) . \\
& \left\{\sum_{j=1}^{n}(-)^{n} \mathcal{S}_{n-j}^{(n)}\left(h^{2}\right) \frac{n ! H_{n-j}(x)}{(n-j) !}(-h)^{j}+(-)^{n} q^{-n(n-1)} H_{n}(x)\right. \\
& \left.+\sum_{j=1}^{\infty}(-)^{n} \mathcal{S}_{n+j}^{(n)}\left(h^{2}\right) \frac{n ! H_{n+j}(x)}{(n+j) !}(-h)^{j}\right\} \cdot<x \mid 0>
\end{aligned}
$$


It is obvious that this expression tends versus the eigenfunctions of the classical harmonic oscillator, as only the central term survives in the limit. The final equation can be compactly written as:

$$
<x\left|n>_{q}=\frac{(-)^{n}}{\sqrt{2^{n}(n)_{q^{-2}} !}} \gamma\left(h^{2}\right) \cdot \sum_{j=-n}^{\infty} \mathcal{S}_{n+j}^{(n)}\left(h^{2}\right) \frac{n ! H_{n+j}(x)}{(n+j) !}(-h)^{|j|} \cdot<x\right| 0>
$$

4.2. $q$-lattice representation. We now consider a representation on the $q$-deformed lattice of the toy-model algebra. As there are several possibilities to realize the $q$-oscillator algebra, we choose the most convenient one for the $q$-position representation:

$$
a_{q}=\frac{1}{\sqrt{1-q^{-2}}} u\left\{u-\xi q^{s-\frac{1}{2}}\right\}
$$

We have put the arbitrary phase angle to 0 , and have chosen $s$ such that for $s=0$ we obtain the undeformed groundstate. The groundstate is given by:

$$
a_{q}\left|0>=0 \rightarrow\left(u-q^{s-\frac{1}{2}} \xi\right)\right| 0>=0 \quad \sum_{n, \pm}\left(u-q^{s-\frac{1}{2}} \xi\right)\left| \pm X_{n}>< \pm X_{n}\right| 0>
$$

Solving for the coefficients, we obtain:

$$
\begin{aligned}
& c_{n}^{ \pm}=< \pm X_{n} \mid 0>=( \pm)^{n} \pi_{0}^{n} q^{\frac{n}{2}} q^{-(n+2 s) \frac{n}{2}} \cdot c_{0} \\
& =( \pm)^{n} \pi_{0}^{n} q^{\frac{s^{2}}{2}} q^{-\frac{(n+s)^{2}}{2}} \cdot q^{\frac{n}{2}} c_{0}
\end{aligned}
$$

For the norm-factor the following summability condition holds:

$$
\sum_{n, \pm}\left|c_{n}^{ \pm}\right|^{2}=1=2 \cdot \sum_{n} \pi_{0}^{2}\left(q^{2 s}\right)^{n} q^{-(n-1) n} \cdot\left|c_{0}\right|^{2}
$$

Which can be solved with the Jacobi triple product identity $[5,6]$ :

$$
\left|c_{0}\right|^{2}=\frac{1}{2\left(q^{-2},-\pi_{0}^{2} q^{2 s},-\pi_{0}^{-2} q^{-2(s+1)} ; q^{-2}\right)_{\infty}}
$$

For the special-case $s=0 ; \pi_{0}=0$ this expression has the compact form:

$$
\left|c_{0}\right|^{2}=\frac{1}{2\left(q^{-2},-1, q^{-2} ; q^{-2}\right)_{\infty}}
$$

The excited states are obtained by the repeated action of $a_{q}$ on the deformed groundstate. This action reads for an arbitrary state $|F\rangle$ :

$$
a_{q}^{*}\left|F>=\frac{1}{\sqrt{1-q^{-2}}} \sum_{ \pm, n}\right| \pm X_{n}>\left\{\left\langle \pm X_{n+2}\left|F>-q^{\bar{s}} q^{n-1} \pi_{0}^{-1}< \pm X_{n+1}\right| F>\right\}\right.
$$

As an example the first excited state is calculated:

$$
\left|1>=\sum_{ \pm, k}\right| \pm X_{k}>\cdot \frac{1}{\sqrt{1-q^{-2}}}\left\{\pi_{0}^{2} q^{-(2 s+1)} q^{-2 k}-q^{s-\bar{s}} q^{-1}\right\} \underbrace{( \pm) \pi_{0}^{k} q^{\frac{k}{2}} q^{-\frac{(k+2 s) k}{2}}}_{< \pm X_{k} \mid 0>}
$$

For a general state we make the following Ansatz:

$$
\left|n>=\sum_{ \pm, k}\right| \pm X_{k}>\frac{1}{\sqrt{\left(q^{-2} ; q^{-2}\right)_{n}}} H_{n}^{q}\left(q^{-2 k}\right)< \pm X_{k} \mid 0>
$$


Here a generalization $H_{n}^{q}$ of the Hermite-polynomials has been introduced. One finds the following recursion formula for $H_{n}^{q}$ :

$$
H_{n}^{q}\left(q^{-2 k}\right)=H_{n-1}^{q}\left(q^{-2(k+2)}\right) q^{-2 k} q^{-(2 s+1)} \pi_{0}^{2}-H_{n-1}^{q}\left(q^{-2(k+1)}\right) q^{\bar{s}-s} q^{-1}
$$

Obviously the factor $q^{-2 k}$ is raising the rank of the polynomial by one. Because of this fact and the knowledge of $H_{0}\left(q^{-2 k}\right)$ one can make the following Ansatz:

$$
H_{n}(z)=\sum_{j=0}^{n} a_{j}^{n} z^{j}
$$

The recursion relation of the $a_{j}^{n}$ suggests yet another substitution to eliminate the $s$-dependencies:

$$
a_{k}^{n}=(-)^{n-k} q^{(n-k)(\bar{s}-s)} q^{-2 k s} \pi_{0}^{2 k} q^{4 k-n} c_{k}^{n}
$$

Those new coefficients fulfill much simpler recursion relations

$$
c_{k}^{n}=q^{-4 k} c_{k-1}^{n-1}+q^{-2 k} c_{k}^{n-1}
$$

which can be solved by:

$$
c_{k}^{n}=\left(q^{-2}\right)^{k(k+1)}\left(\begin{array}{l}
n \\
k
\end{array}\right)_{q^{-2}}
$$

Thus obtaining the final result:

$$
a_{k}^{n}=(-)^{n-k} \pi_{0}^{2 k} q^{(n-k)(\bar{s}-s)} q^{-2 k(k-1+s)} q^{-n}\left(\begin{array}{l}
n \\
k
\end{array}\right)_{q^{-2}}
$$

We have therefore proven the following theorem of $q$-analysis:

$$
\sum_{k=-\infty}^{\infty} \frac{H_{n}^{q}\left(q^{-2 k}\right) H_{n^{\prime}}^{q}\left(q^{-2 k}\right)}{\sqrt{\left(q^{-2} ; q^{-2}\right)_{n}} \sqrt{\left(q^{-2} ; q^{-2}\right)_{n}^{\prime}}} \overbrace{\left|< \pm X_{k}\right| 0>\left.\right|^{2}}^{:=\frac{\mu_{k}}{2} c_{0}^{2}=\pi_{0}^{2 k} q^{k} q^{-(k+2 s) k} c_{0}^{2}}=\delta_{n, n^{\prime}}
$$

Having introduced the measure $\mu_{k}$ we compactly write the expression:

$$
\begin{aligned}
& \sum_{k=-\infty}^{\infty} \mu_{k} H_{n}^{q}\left(q^{-2 k}\right) H_{n^{\prime}}^{q}\left(q^{-2 k}\right)=\left(q^{-2} ; q^{-2}\right)_{n} \delta_{n, n^{\prime}} \\
& \mu_{k}=\frac{\pi_{0}^{2 k} q^{k} q^{-(k+2 s) k}}{\left(q^{-2},-\pi_{0}^{2} q^{2 s},-\pi_{0}^{-2} q^{-2(s+1)} ; q^{-2}\right)_{\infty}} \quad H_{n}^{q}(z)=\sum_{j=0}^{n} a_{j}^{n} z^{j}
\end{aligned}
$$

As in the undeformed case the polynomials for $s \neq 0$ are obtained from those for $s=0$ by the action of a unitary operator $\hat{\mathcal{D}}(s)$. But on the $q$-lattice this fact is less useful as the operator only formally exist for most values of s. Using the expression for $\hat{\mathcal{D}}(s)$ one immediately finds:

$$
\hat{\mathcal{D}}(s)=e^{\frac{h}{\sqrt{2}}\left(a \bar{s}-a^{*} s\right)}=\xi^{-i s_{2}} u^{-s_{1}} q^{-\frac{s_{1} s_{2}}{2}}
$$

Algebraically one finds: $\hat{\mathcal{D}}(s) a_{q}(s=0)=a_{q}(s) \hat{\mathcal{D}}(s)$. The action of $\hat{\mathcal{D}}(s)$ on the groundstate is also computable. The defining relation for the groundstate $a_{q} \mid 0>_{q}=0$ reads:

$$
\left(u-\xi q^{\frac{1}{2}}\right)\left|0>_{q}^{s=0}=0 \quad \rightarrow u\right| 0>_{q}^{s=0}=q^{-\frac{1}{2}} \xi \mid 0>_{q}^{s=0}
$$


This allows to define the action of any power of $u$ on the groundstate by $u^{z} \mid 0>_{q}^{s=0}:=$ $q^{-\frac{z}{2}} \xi^{z} \mid 0>_{q}^{s=0}$. Using this one finally obtains:

$$
\hat{\mathcal{D}}(s)\left|0>_{q}^{s=0}=\xi^{-s}\right| 0>_{q}^{s=0} q^{-\frac{s_{1}\left(s_{2}+1\right)}{2}} \propto \mid 0>_{q}^{s}
$$

The proportionality holds because a factor $q^{-s n}$ is generated on the lattice, which gives the s-dependence of the groundstate.

5. Relation to $q$-hypergeometric functions. The polynomials that were obtained in the previous section from deformation theory of the Heisenberg algebra, can be expressed in the language of $q$-hypergeometric functions and identified with well known generalizations of the classical Hermite polynomials. Therefore it is once more possible to make contact between the deformation theory of Lie-algebra and the theory of $q$-polynomials [4]. Those generalized $q$-Hermite polynomials appear now as the spectrum of the Hamiltonian of a suitable $q$-deformation of the Heisenberg algebra, realized as essentially self-adjoint operators on a Hilbert space.

Depending on the Hilbert space representation continuous (real line) or discrete (q-lattice) $q$-polynomials are found. Those can be interpreted as quantum-mechanical single-particle wave functions in a $q$-deformed oscillator potential. The two relevant cases will now be investigated:

5.1. q-hypergeometric polynomials. The general definition of a $q$-hypergeometric polynomial is recalled: A basic hypergeometric series or q-hypergeometric series is defined by $[4,5,6]$ :

$$
\begin{aligned}
{ }_{r} \Phi_{s}\left(\begin{array}{c|c}
a_{1}, \ldots, a_{r} \\
b_{1}, \ldots, b_{s}
\end{array}\right. & q, z)={ }_{r} \Phi_{s}\left(a_{1}, \ldots, a_{r}, b_{1}, \ldots, b_{s} ; q, z\right) \\
: & =\sum_{k=0}^{\infty} \frac{\left(a_{1}, \ldots, a_{r} ; q\right)_{k}}{\left(b_{1}, \ldots, b_{s}, q ; q\right)_{k}}\left((-1)^{k} q^{k(k-1) / 2}\right)^{1+s-r} z^{k}, \quad r, s \in \mathbb{Z}
\end{aligned}
$$

here the $q$-shifted factorial is used again:

$$
(a ; q)_{0}:=1 \quad \text { and } \quad(a ; q)_{k}:=\prod_{n=0}^{k-1}\left(1-a q^{n}\right)
$$

with the following convenient notation:

$$
\left(a_{1}, a_{2}, \ldots, a_{r} ; q\right)_{k}:=\left(a_{1} ; q\right)_{k}\left(a_{2} ; q\right)_{k} \ldots\left(a_{r} ; q\right)_{k}, \quad k=0,1,2, \ldots, \infty
$$

Those $q$-hypergeometric series have well known properties and have extensively been studied in the literature $[4,5,6]$.

5.2. Stieltjes Wigert polynomials. The Stieltjes Wigert polynomials are a well known generalization of the classical Hermite polynomials. They are defined as:

The Stieltjes Wigert Polynomial

$$
S_{n}(x ; q):=\frac{1}{(q ; q)_{n}}{ }_{1} \Phi_{1}\left(\begin{array}{c|c}
q^{-n} & q ;-x q^{n+1} \\
0 &
\end{array}\right)
$$


The solution on the $q$-lattice can be rewritten as a basic hypergeometric function:

$$
H_{n}^{q}(z):=(-)^{n} q^{i n s^{\prime \prime}} q^{\prime \frac{n}{2}}{ }_{1} \Phi_{1}\left(\begin{array}{c|c}
q^{\prime-n} & q^{\prime} ;-z q^{\prime n+s^{\prime}}
\end{array}\right)
$$

One has $s=s^{\prime}+i s^{\prime \prime}$ and for notational convenience $q^{\prime}:=q^{-2}$ was introduced (in books on basic hypergeometric functions one usually has the convention $0<q<1$ ). Comparison of the two results shows that except for trivial factors and a translation of the argument the wave functions on the $q$-lattice are the Stieltjes Wigert polynomial. It is well known that those functions have the following limit behavior for $q \rightarrow 1$ and $x \rightarrow q^{-1} x \sqrt{2(1-q)}+1$ :

$$
\lim _{q \uparrow 1} \frac{(q ; q)_{n} \mathcal{S}_{n}\left(q^{-1} x \sqrt{2(1-q)}+1 ; q\right)}{\left(\frac{1-q}{2}\right)^{\frac{n}{2}}}=(-1)^{n} H_{n}(x)
$$

This behavior ensures that a suitable limit from the $q$-lattice to the continuous case can be defined, such that the $q$-deformed wave functions will tend to the solutions of the classical oscillator.

In the $q$-oscillator representation on the classical phase space, a similar result is obtained (using $s=0$ for convenience).

$$
<x \mid n>_{q}=(-)^{n} \frac{q^{\frac{n}{2}}}{\sqrt{\left(q^{\prime} ; q^{\prime}\right)_{n}}}{ }_{1} \Phi_{1}\left(\begin{array}{c}
q^{\prime-n} \\
0
\end{array} \mid q^{\prime} ; e^{-2 h x} q^{\prime n+\frac{1}{2}}\right)
$$

Here the limit was already established by the explicit expansion $(33)$ in $h=\sqrt{\log (q)}$. Now one sees that as $q \rightarrow 1$ both solutions get more and more similar $\left(^{6}\right)$, in fact (61) becomes an interpolating function for (59) on the lattice points (which become more and more densely spaced). This is what one expects a the $q$-lattice is supposed to be a deformation of the classical real-line, so that both representations should become more and more similar as $q$ tends to 1 .

The physical representation is certainly (59) as here the $q$-deformation of the Heisenberg algebra is reflected in both the Hamiltonian (in physical words the potential) and the representation of space time (which is in this modest example the real line). The continuous example is an amusing example how the classical wave functions can be smoothly deformed, but it is irrelevant for generalizations in a more physical setting as the $S O_{q}(3)$ or $E_{q}(3)$ symmetry of the Euclidean space [10].

6. Summary and outlook. The well known Stieltjes Wigert polynomials were shown to stem from the representation theory of the $q$-Heisenberg algebra. They appear as wavefunctions for one particle states in a $q$-oscillator potential, which is expressed in the generators of the $q$-deformed phase space. The oscillator variables were constructed by the concatenation of embeddings of the respective algebras. This process is not unique in one dimension, and it is tempting to study several realizations of the problem, leading to different sets of $q$-polynomials (and examples of $q$-Fourier transforms). By postulating

$\left({ }^{6}\right)$ One has to keep in mind that the factor $\frac{1}{\sqrt{\left(q^{-2} ; q^{-2}\right)_{n}}}$ has been taken out by the definition of $H_{n}^{q}$. 
invariance under $q$-Fourier transformation (which is valid for $q=1$ ) one might be able to find a natural and hopefully unique solution to the problem.

It will be of interest to study the problem in higher dimensions, as the richer (i.e. Hopf) structure of the phase space for $N \geq 3$, will restrict the set of solutions significantly, so that a unique solution might be possible.

A three dimensional generalization could be relevant to solid state physics, and provide a basis for quantitative predictions.

\section{References}

[1] J. Schwenk, J. Wess, A Quantum Mechanical toy model, Phys. Lett., B 291 (1992) 273.

[2] A. Hebecker, S. Schreckenberg, J. Schwenk, W. Weich, J. Wess, Representations of a q-deformed Heisenberg Algebra, MPI-Ph/93-45, (1993).

[3] M. Fichtmueller, A. Lorek and J. Wess, Q-deformed Phase Space and its Lattice Structure, MPI-PhT/95-109.

[4] Tom H. Koornwinder, Orthogonal Polynomials in Connection with Quantum Groups, P. Nevai (ed.), Orthogonal Polynomials,Kluwer Academic Publishers, (1990) 257-292.

[5] Roelof Koekoek and René F. Swarttouw, The Askey-scheme of hypergeometric orthogonal polynomials and its q-analogue, Delft University of Technology, Reports of the faculty of technical Mathematics and Informatics no 94-05, (1994).

[6] Gasper and Rahman, Basic Hypergeometric Series, Cambridge University Press, (1990).

[7] A. Macfarlane, On q-analogues of the quantum harmonic oscillator and the quantum group $S U_{q}(2)$, J. Phys., A 22 (1989) 4581.

[8] P. P. Kulish, On Recent Progress in Quantum Groups an introductory review, Jahrbuch Überblicke Mathematik 1993, Vieweg, (1993) 97.

[9] T. Curtwright, C. Zachos, Paradigms of Quantum Algebras, ANL-HEP-PR-90-61, (1990).

[10] Gaetano Fiore, The $S_{q}(N, \mathbb{R})$-Symmetric Harmonic Oscillator on the Quantum Euclidean Space $\mathbb{R}_{q}^{N}$ and It's Hilbert Space Structure, International Journal of Modern Physics, Vol. 8, 26 (1993) 4679-4729.

[11] Joachim Seifert, Quantum Mechanical Representations of the Q-Oscillator, forthcoming publication. 